\title{
BL Lac Objects
}

\section{Marco Bondi*}

INAF-IRA, Via Gobetti 101, 40129 Bologna, Italy

E-mail: bondi@ira.inaf.it

BL Lac objects have been a favourite target for VLBI observations since the beginnings of this technique. I will review some of the recent results obtained studying BL Lac objects on parsec scale. In particular, I will cover the following points: 1) new sample of BL Lac objects; 2) the pc-scale kinematics in the TeV BL Lac objects; 3) coordinated radio and broadband (optical, Xray, $\gamma$-ray) observations. Finally, I will briefly introduce the AGILE mission and its contribution to this topic.

The 8th European VLBI Network Symposium

September 26-29, 2006

Toruń, Poland

${ }^{*}$ Speaker. 


\section{Introduction}

The emission from BL Lac objects is dominated by a non-thermal continuum extending from the radio to $\gamma$-ray band and in a few cases to the TeV region of the electromagnetic spectrum. Typical observational properties of these objects are as follows: high luminosity, a flat radio spectrum that steepens in the optical-IR bands, relatively high optical and radio polarisation, rapid variability in at least one of the radio, optical or X-ray bands, and weak or absent emission lines (see [1], [2] for extensive reviews on BL Lac objects). These properties have been interpreted in terms of a relativistic jet aligned to the line of sight [3], implying the existence of a parent population of objects oriented at large angles to the line of sight and identified with the low-luminosity edge-darkened

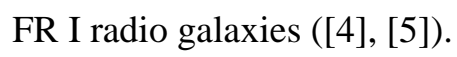

In this review, I will focus on some recent developments of the research on BL Lac objects which took advantage of VLBI observations. Some topics are deliberately skipped because they are the subject of other reviews presented at this conference (e.g. polarisation properties are extensively covered in D. Gabuzda's review [6] and pc-scale properties of the radio jets are discussed in A. Lobanov's review [7]).

\section{New samples of BL Lac objects}

Almost 10 years ago, Fossati and collaborators [8] introduced the "blazar sequence". After the authors had collected broadband information on BL Lac objects and flat-spectrum radio quasars from 3 main samples, they showed that there was a remarkable anticorrelation between the peak frequency of the synchrotron component and the radio luminosity: higher-luminosity objects peaked at lower frequency and lower-luminosity objects peaked at higher frequency. An intriguing physical explanation of this behaviour was proposed by Ghisellini and collaborators [9], who interpreted this trend as produced by increasing radiative losses in the higher-luminosity objects. Unfortunately, the samples used by Fossati were highly biased and, as noted by Caccianiga \& Marchã [10], the remarkable continuity showed by the "blazar sequence" was, at least for the lowluminosity objects, due to selection effects, not physical. In particular, the lack of low-luminosity objects with steep radio to X-ray spectral index $\left(\alpha_{\mathrm{RX}}\right)$ in the samples used by Fossati, could simply be explained as due to selection effects.

In order to have a full view of the BL Lac broadband properties, new samples of objects covering more homogeneously the plane $\alpha_{\mathrm{RX}}$ and radio luminosity are necessary. This is the goal of at least two projects developed in the last years: the Class Blazar Survey [10], and the DXBRS BL Lac Sample. The latter is presented in the contribution of H. Bignall [11]. The motivation behind the Class Blazars Sample is to look for low X-ray emitting BL Lacs in a sample of about 300 flat-spectrum radio sources. Radio observations are essential to establish the compactness of the sources and to separate the BL Lac objects from possible contaminating sources. From their study, Caccianiga \& Marchã have found that a significant and large fraction $(\sim 30 \%)$ of lowluminosity blazars have $\alpha_{\mathrm{RX}}>0.75$ and lie outside the "blazar sequence". This result shows the importance of a correct and unbiased sampling of the low-power regime of the blazar population.

Another interesting recent paper by Giroletti and collaborators [12] presents a sample of nearby $(z<0.3)$ BL Lacs with HST archive data and VLBI observations. 


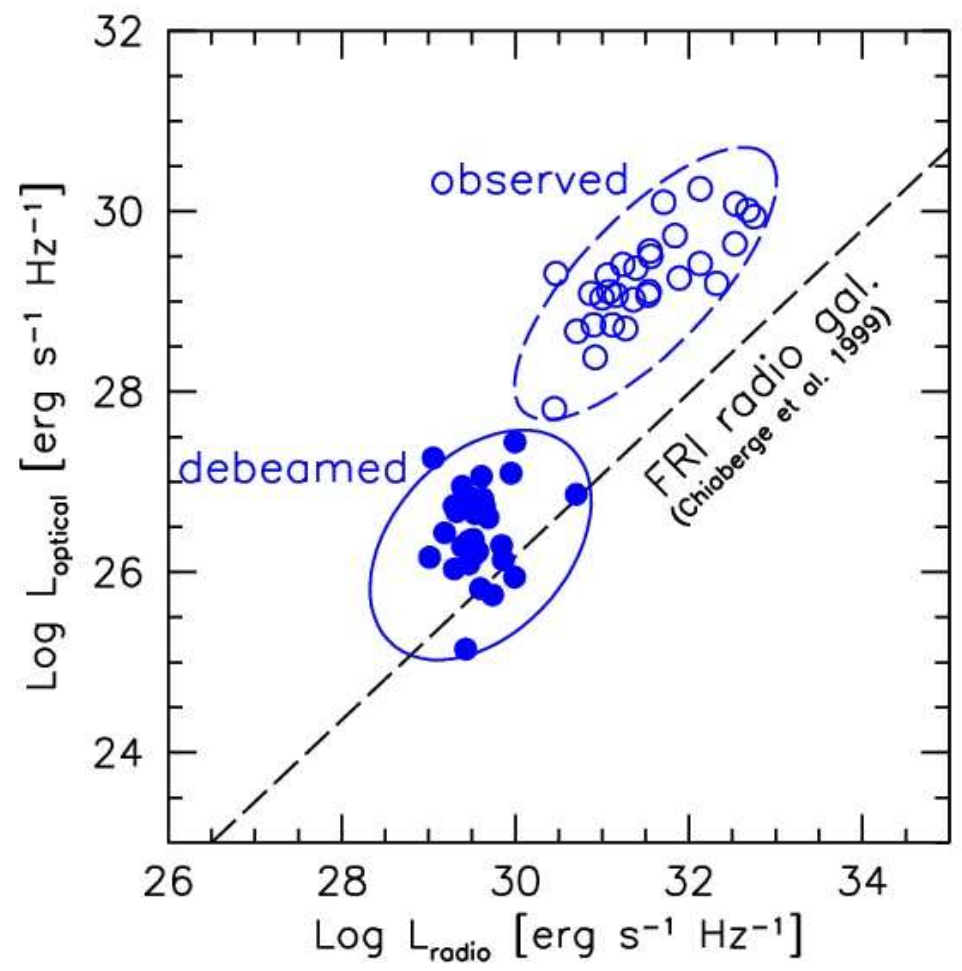

Figure 1: Optical vs radio core luminosity for BL Lac objects in the Giroletti's sample. Open dots are observed luminosities, filled ones are luminosities corrected for Doppler beaming assuming a viewing angle of $60^{\circ}$. The dashed line shows the radio-optical correlation found for cores of FR I radio galaxies (Chiaberge et al. 1999).

HST observations allow to separate the compact core emitting non-thermal synchrotron emission from the galaxy thermal emission and to compare the optical and radio (VLBI) properties of the core. Giroletti and collaborators have found (see Fig. 1) that the radio and optical nuclear luminosity are correlated in BL Lac objects and follow the same trend observed in low-power (FR I) radio galaxies [13], but the optical cores of BL Lacs are about two orders of magnitude brighter than those of FR I radio galaxies with the same core radio luminosity. Using the VLBI observations of the BL Lac objects in the sample, it is possible to derive their beaming parameters and recalculate the core luminosity assuming a viewing angle of $60^{\circ}$, i.e. the average angle for an unbeamed radio galaxy. The de-beamed BL Lac objects move to lower luminosities and fit to the correlation found for FR I radio galaxies (see Fig. 1). This result is a strong confirmation that the origin of the radio and optical nuclear emission is the same (a synchrotron-emitting compact core) and supports the unified-scheme scenario, in which FR I radio galaxies are the parent population of BL Lac objects.

\section{Jet-speed in TeV BL Lac objects}

The observed jet-speeds in BL Lac objects have a wide range of values. Recently, Jorstad and collaborators [14] derived the jet-speed distribution of BL Lac objects, radio galaxies and quasars from 7-mm observations spanned over 17 epochs. BL Lacs are found to have a flat jet-speed 

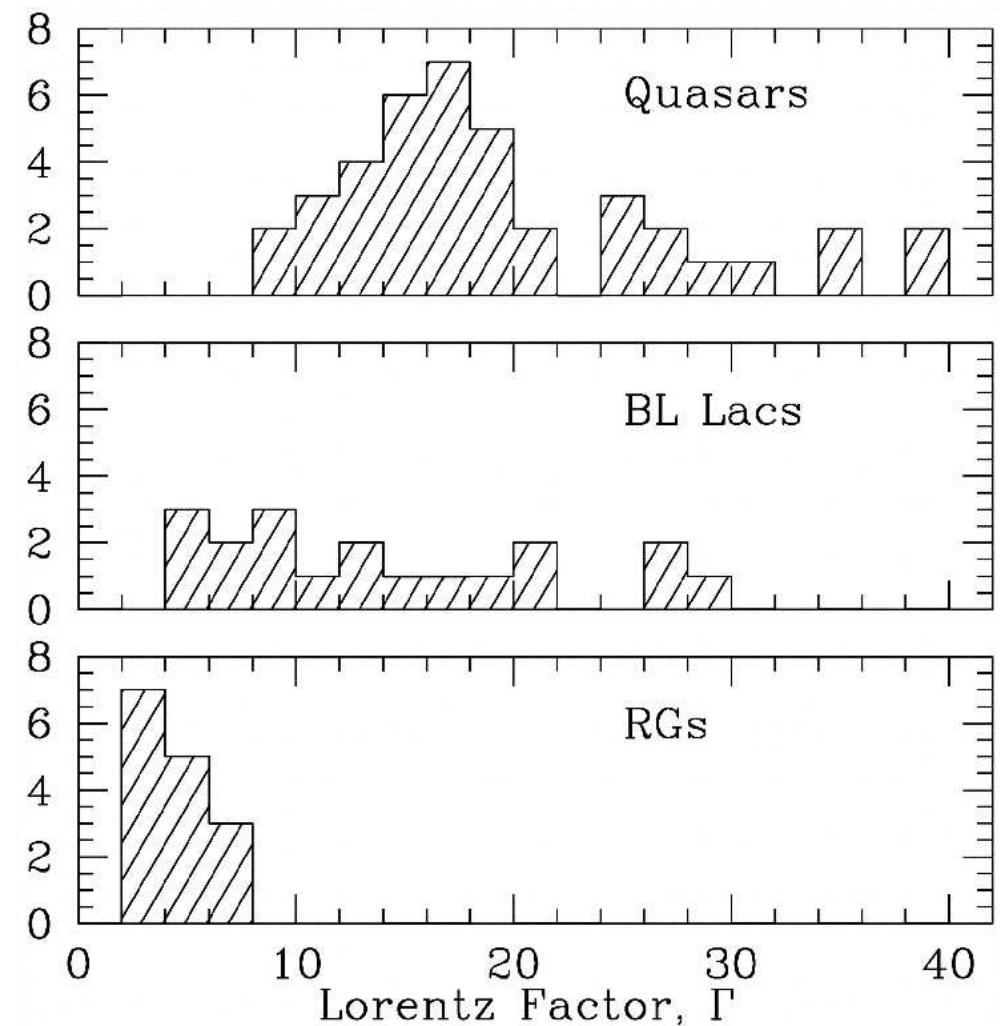

Figure 2: Distribution of Lorentz factors of superluminal jet components in 15 AGNs derived from 17 epochs of observations at $7 \mathrm{~mm}$ wavelength. From Jorstad et al. 2005.

distribution with values of $\Gamma$ from a few to as high as 30 (see Fig. 2).

Piner \& Edwards [15] observed $3 \mathrm{TeV}$ BL Lacs at $15 \mathrm{GHz}$ with the VLBA at three/four epochs spanning 1 year and reached the following conclusions: 1) the jet morphologies are very similar: a collimated region, followed by a pronounced bending and the transition to a diffuse, less collimated jet beyond the bending (see also [16]); 2) the pc-scale components in TeV BL Lacs are predominantly stationary or subluminal, $\Gamma \simeq 2-4$, (see Fig. 3). Similar jet morphologies and velocities have been found in the jet of Mrk 501 [17], another TeV BL Lac. So there seems to be a consistent behaviour for the kinematically-derived Lorentz factors in this particular class of BL Lac objects that is in contrast with the Lorentz factors invoked to explain the $\gamma$-ray emission, which require $\Gamma>10$.

Two families of models have been proposed to explain this discrepancy:

- Velocity profile in cylindrical jets. The jet can have a radial velocity profile, e.g. a strong deceleration along the jet [18] or a transverse velocity profile, e.g. a faster spine and a slower layer or sheath [19]. In both cases one region of the jet sees the beamed radiation from the other region producing enhanced $\gamma$-ray emission (Fig. 4). The spine-sheath model can also explain the limb-brightened morphology of the inner jet in Mrk 501 [17].

- The VLBI jet is modelled as a conical jet with a finite opening angle and constant velocity. In this case, the observed apparent velocity and flux-boosting factor need to be calculated 


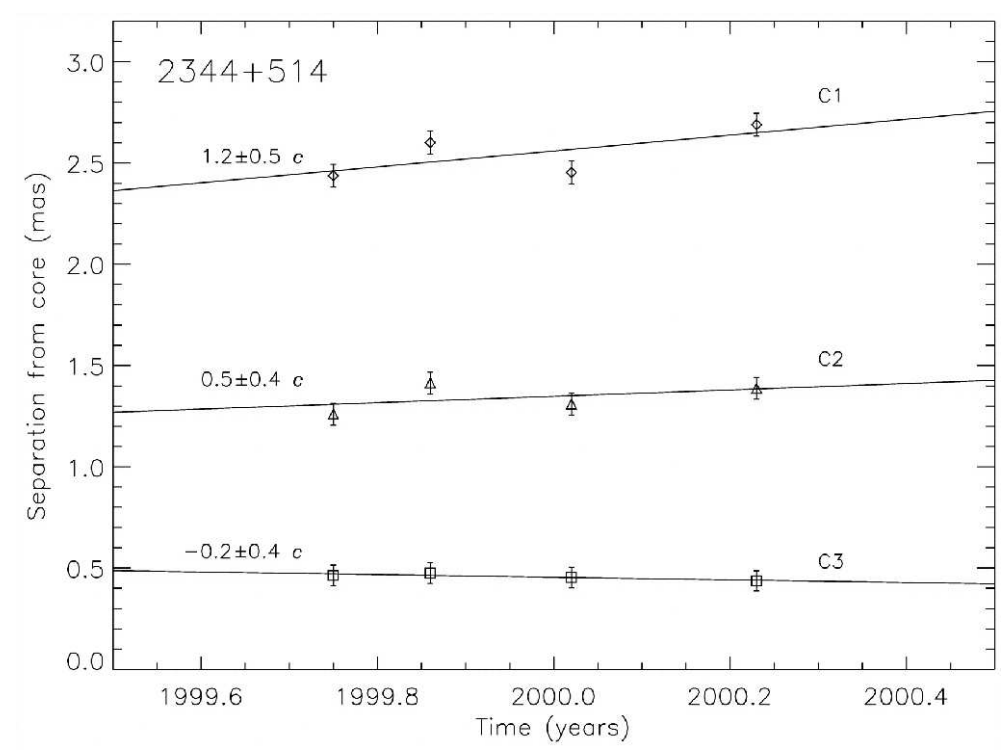

Figure 3: Distances from the core of fitted Gaussian component as a function of time for the TeV BL Lac object 2344+514 observed at 15 GHz. From Piner \& Edwards 2004.

integrating over the angular distributions of these quantities across the width of the jet. The resulting apparent speed, for high bulk Lorentz factor $\Gamma>10$, can be much lower than those obtained assuming a cylindrical geometry for the jet and thus producing subluminal or mildly relativistic jet features (Fig. 5) [20].

\section{Coordinated multi-wavelength campaigns}

Recently, several papers presented results from coordinated campaigns on few well-known BL Lac objects with the general goal of studying the possible correlation between the radio and higher frequency (optical, X-ray, $\gamma$-ray) variability ([21],[22],[23]). These campaigns took great advantage of the Whole-Earth Blazar Telescope (WEBT) collaboration, a network of optical and radio telescopes that, together, are able to obtain high temporal density monitoring of blazars. A common result of these studies was that, while the low-level optical variability was not well correlated with that in the radio domain, the major optical bursts were followed by radio fluxdensity and polarisation variations in the VLBI core, with no apparent changes in the VLBI jet (see also [24]).

In particular, Bach and collaborators [23] have presented the results from a 10-year optical and radio (both single-dish and VLBI) monitoring of BL Lac itself. The authors stress the importance of the VLBI monitoring that allows to disentangle the different components contributing to the singledish radio variability. They have found that the radio flux-density variability in the single-dish data is not necessarily a good tracer of the VLBI core or optical variability, while the spectral variability in the single-dish data follows the variations of the VLBI core. So, multi-wavelength single-dish observations can be used to fill in the gaps in the VLBI monitoring to trace the variations of the VLBI core and investigate the correlation with optical variability. Monitoring of BL Lac reveals a 


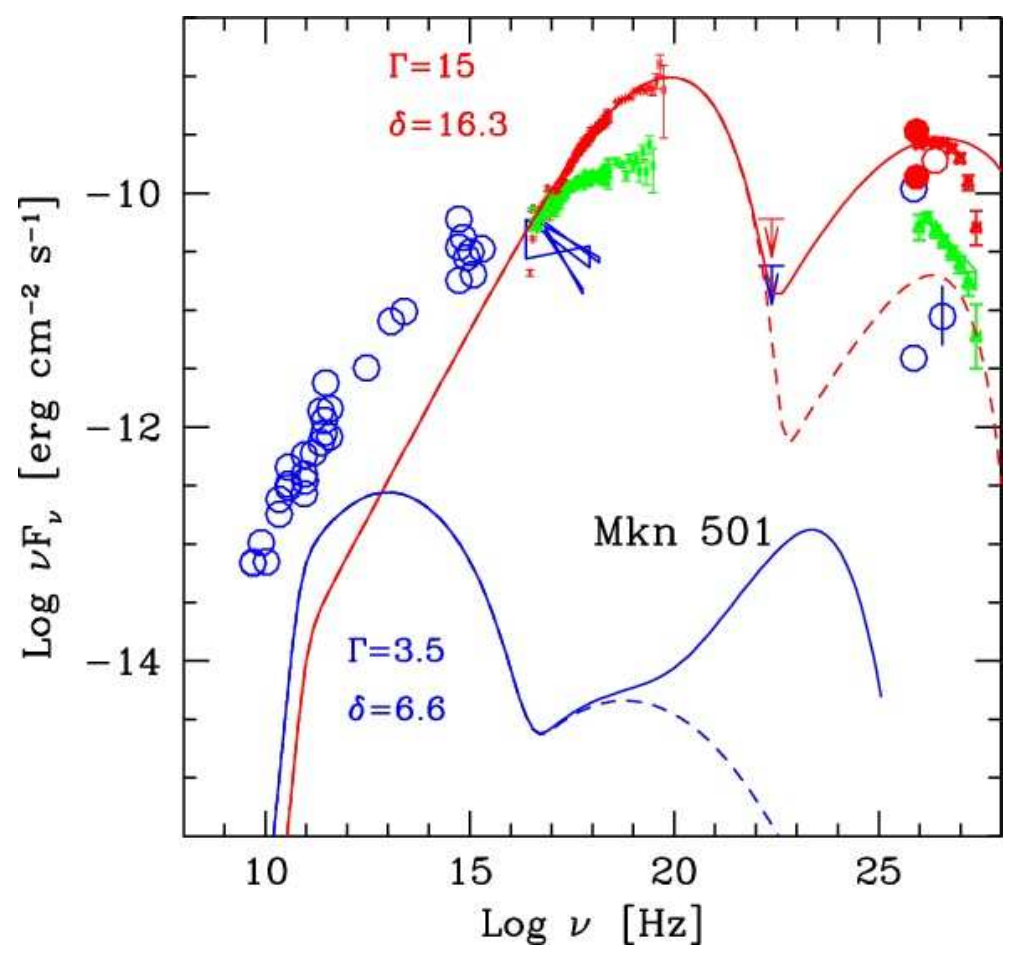

Figure 4: Example of SED produced by the spine-sheath model. Emission from the slower sheath is in blue and that from the spine is in red. Dashed lines correspond to the emission of the spine (sheath) without taking into account the seed photons coming from the sheath (spine). From Ghisellini et al. 2005, see the paper for details of the model.

fair correlation between the optical and radio bands with time delay ranging from 50 to about 150 days, depending on the frequency of the radio data (as expected, shorter time delays are at higher radio frequencies).

The connection between $\gamma$-ray outbursts and the ejection of VLBI components was found and studied in the past ([25], [26], [27]). In particular, the $\gamma$-ray flares are found coincident with the extrapolated epoch of zero separation from the core of a superluminal radio component.

Charlot and collaborators [28] searched for correlated radio and $\mathrm{TeV}$ variability in the $\mathrm{TeV}$ BL Lac object Mkn 421. The VLBI observations were carried out at three closely spaced epochs (one month apart) and the source was also monitored in the $\mathrm{TeV}$ region during the same period. The VLBI jet showed no evidence of variability, while there was evidence of variations in both the total and polarized flux of the VLBI core and in the $\mathrm{TeV}$ emission. The data suggest that the $\mathrm{TeV}$ emission and the VLBI core variability can be connected.

\section{Future}

The systematic study of a larger number of objects in the radio, optical, X-ray and $\gamma$-ray window is mandatory to understand any class of radio sources and this is even more true for BL Lacs as they are clearly multiwavelength objects. The results obtained by the WEBT collaboration 

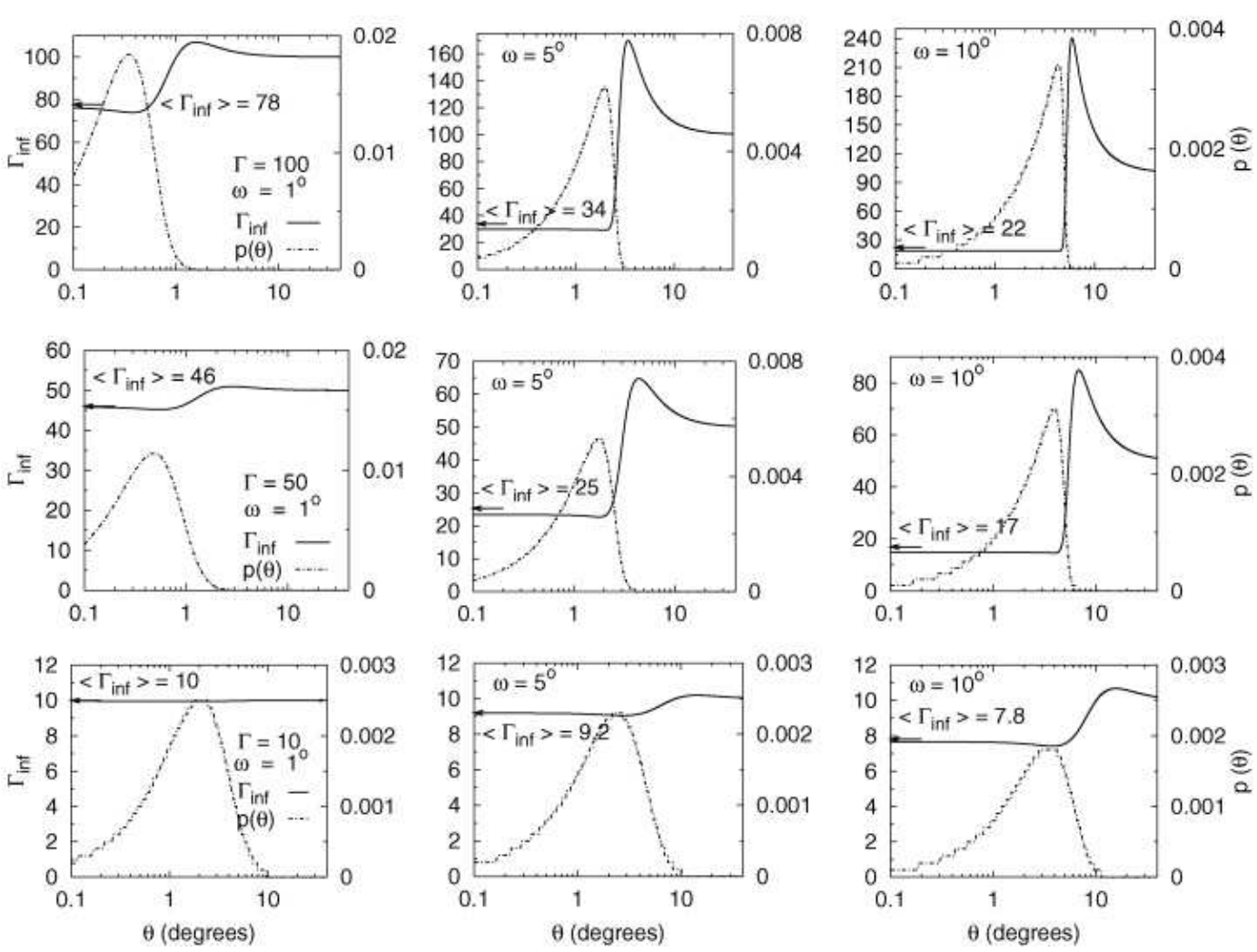

Figure 5: Inferred Lorentz factors, $\Gamma_{\text {inf }}$, (solid curves) vs. the jet viewing angle, $\theta$. The top row has $\Gamma=100$, the middle row $\Gamma=50$ and the bottom row $\Gamma=10$. Each panel is labelled with the actual value of the full opening angle $\omega$. The expected averaged measured vales are also given. From Gopal-Krishna et al. 2006.

are very encouraging and a more complete monitoring will be achieved once AGILE and GLAST will be launched and operating.

AGILE (a full description of the technical characteristics and scientific case can be found on the AGILE Web page: http://agile.rm.iasf.cnr.it) is a space scientific mission built by a consortium of Italian agencies, universities and companies. The instrumentation on board will allow imaging in the $\gamma$-ray (in the range $30 \mathrm{MeV}-30 \mathrm{GeV}$ ) and in the hard $\mathrm{X}$-ray band (in the range $15 \mathrm{keV}$ $-45 \mathrm{keV}$ ) and the launch is scheduled for April 2007. The monitoring of AGNs is one of the key science projects of AGILE and its characteristics are well suited for this task. AGILE will have a source location determination of 5-20 arcmin (about twice better than EGRET), a on-axis sensitivity comparable to EGRET and off-axis sensitivity better than EGRET, a field of view of 3 sr about 6 times that of EGRET, and a sensitivity for a 1-year all sky survey 3 times better than EGRET.

Even if the angular resolution of VLBI is at least two orders of magnitude better than those reachable at optical, X-ray or $\gamma$-ray wavelengths, the broadband correlated campaigns have shown that the radiation emitted in these bands is originating in an even more compact region than that accessible to cm-wave VLBI. The millimeter-VLBI array [29] and the next generation of VLBI space interferometry [30] can help closing this gap and we will be able to investigate the same physical region from radio to $\gamma$-rays in the near future. 


\section{References}

[1] K.I. Kollgaard 1994, Vistas in Astronomy, 38, 29

[2] C.M. Urry \& P. Padovani 1995, PASP, 107, 715

[3] R. Blandford \& M.J. Rees 1978, in Proc. Pittsburgh Conf. on BL Lac Objects, ed. A.N. Wolfe, p.328

[4] I.W.A. Browne 1983, MNRAS, 204, 23

[5] J.F.C. Wardle et al. 1984, ApJ, 279,93

[6] D. Gabuzda 2006, PoS (8thEVN) 011

[7] A. Lobanov 2006, $\operatorname{PoS}(8 \mathrm{thEVN}) 003$

[8] G. Fossati et al. 1998, MNRAS, 299, 433

[9] G. Ghisellini et al. 1998, MNRAS, 301, 451

[10] A. Caccianiga \& M.J.M. Marchã 2004, MNRAS, 348, 937

[11] H. Bignall 2006, PoS (8thEVN) 026

[12] M. Giroletti et al. 2006, ApJ, 646, 801

[13] M. Chiaberge et al. 1999, $A \& A, 349,77$

[14] S.G. Jorstad et al. 2005, AJ, 130, 1418

[15] B.G. Piner \& P.G. Edwards 2004, ApJ, 600, 115

[16] T.A. Rector et al. 2003, AJ, 125, 1060

[17] M. Giroletti et al. 2004, ApJ, 600, 127

[18] M. Georganopoulos \& D. Kazanas 2003, ApJ, 594, L27

[19] G. Ghisellini et al. 2005, $A \& A, 432,401$

[20] Gopal-Krishna et al. 2004, ApJ, 615, L81

[21] M. Böttcher et al. 2005, ApJ, 631, 139

[22] C.M. Raitieri et al. 2005, $A \& A, 438,39$

[23] U. Bach et al. 2006, $A \& A, 456,105$

[24] E. Massaro et al. 2004, A\&A, 423, 935

[25] M. Pohl et al. 1995, A\&A, 303, 383

[26] S.G. Jorstad et al. 2001, ApJ, 556, 738

[27] A. Lähtenmäki \& Valtaoja 2003, ApJ, 590, 95

[28] P. Charlot et al. 2006, $A \& A, 457455$

[29] T. Krichbaum 2006, PoS (8thEVN) 002

[30] H. Hirabayashi 2006, PoS (8thEVN) 062 